\title{
A note on the hardness results for the labeled perfect matching problems in bipartite graphs
}

\author{
Jérôme Monnot*
}

September 28, 2007

\begin{abstract}
In this note, we strengthen the inapproximation bound of $O(\log n)$ for the labeled perfect matching problem established in J. Monnot, The Labeled perfect matching in bipartite graphs, Information Processing Letters 96 (2005) 81-88, using a self improving operation in some hard instances. It is interesting to note that this self improving operation does not work for all instances. Moreover, based on this approach we deduce that the problem does not admit constant approximation algorithms for connected planar cubic bipartite graphs.
\end{abstract}

Keywords: labeled matching; bipartite graphs; approximation and complexity; inapproximation bounds.

\section{Introduction}

A matching $M$ on a graph $G=(V, E)$ is a subset of edges that are pairwise non adjacent; $M$ is said perfect if it covers the vertex set $V$ of $G$. In the labeled perfect matching problem (LABELED Min PM in short), we are given a simple graph $G=(V, E)$ on $|V|=2 n$ vertices which contains a perfect matching together with a color (or label) function $\mathcal{L}$ : $E \rightarrow\left\{c_{0}, \ldots, c_{q}\right\}$ on the edge set of $G$. For $i=0, \ldots, q$, we denote by $\mathcal{L}_{i} \subseteq E$ the set of edges of color $c_{i}$. The goal of LABELED Min PM is to find a perfect matching on $G$ that uses a minimum number of colors. Alternatively, if $G\left[\mathcal{L}^{\prime}\right]=\left(V, E^{\prime}\right)$ where $E^{\prime}=\{e \in E$ : $\left.\mathcal{L}(e) \in \mathcal{L}^{\prime}\right\}$ denotes the subgraph induced by the edges of colors $\mathcal{L}^{\prime} \subseteq\left\{c_{0}, \ldots, c_{q}\right\}$, then LABELED Min $P M$ aims at finding a subset $\mathcal{L}^{\prime}$ of minimum size such that $G\left[\mathcal{L}^{\prime}\right]$ contains a perfect matching. In [9] a generalization, called perfect matching under categorization, has been studied. In this framework, each edge $e$ has also a non-negative weight $w(e)$, and the colors are called categories (thus, $q+1$ indicates the number of categories). The goal is to find a perfect matching $M$ of $E$ minimizing $\sum_{i=0}^{q} \max _{e \in \mathcal{L}_{i} \cap M} w(e)$. In [9], it is shown that, on the one hand, the problem is polynomial when the number of categories (i.e., colors) is fixed, and on the other hand, the problem is NP-hard when the weights take values 0 or 1 and the graph is a collection of disjoint 4-cycles. Note that the case $w(e)=1, \forall e \in E$ corresponds to LABELED Min PM. Very recently, some approximation results are obtained for LABELED Min PM when the graphs are bipartite 2-regular or complete bipartite $K_{n, n}$, [7]. In particular, it is shown that the 2-regular bipartite case is

* CNRS LAMSADE , Université Paris-Dauphine, Place du Maréchal De Lattre de Tassigny, F-75775 Paris Cedex 16, France. E-mail: monnot@lamsade.dauphine.fr 
equivalent to the minimum satisfiability problem, and that a greedy algorithm picking at each iteration a monocolored matching of maximum size provides a $\frac{r+H_{r}}{2}$-approximation in complete bipartite graphs where $r$ is the maximum of times that a color appears in the graph and $H_{r}$ is the $r$-th harmonic number. Moreover, it is proved that LABELED Min $P M$ is not $O(\log n)$-approximable in bipartite complete graphs. In [6], this problem is motivated by some applications in timetable problems. Several related works concerning some matching problems on colored graphs can be found in $[3,4,5]$

In this note, we prove first that LABELED Min PM is not in APX whenever the bipartite graphs have a maximum degree of 3 . Hence, there is a gap of approximability between graphs of maximum degree 2 and 3 since we can easily deduce from [7] that LABELED Min PM is 2-approximable in bipartite graphs of maximum degree 2 . Using a weaker complexity hypothesis, we can even obtain that LABELED Min PM is not $2^{O\left(\log ^{1-\varepsilon} n\right)}$-approximable in bipartite graphs of maximum degree 3 on $n$ vertices, unless NP

$\subseteq$ DTIME $\left(2^{O\left(\log ^{1 / \varepsilon} n\right)}\right)$. Dealing with the unbounded degree case, this yields to the fact that LABELED Min PM is not in polyLog-APX, unless $\mathbf{P}=\mathbf{N P}$.

In the following, given an instance $I$, we denote by $\operatorname{opt}(I)$ and $\operatorname{apx}(I)$ the value of an optimal and an approximate solution, respectively for LABELED Min PM. We say that an algorithm $\mathcal{A}$ is a $\rho$-approximation (with $\rho \geq 1$ ) if $\operatorname{apx}(I) \leq \rho \times \operatorname{opt}(I)$ for any instance $I$.

Finally, in order to simplify the proofs exposed in the rest of the paper, the results concern a variation of LABELED Min PM, where the value of each perfect matching $M$ is given by $\operatorname{val}_{1}(M)=\operatorname{val}(M)-1$. This problem is denoted LABELED Min PM 1 and we have for any instance $I, \operatorname{apx}_{1}(I)=\operatorname{apx}(I)-1$ and $\operatorname{opt}_{1}(I)=\operatorname{opt}(I)-1$. It is important to note that a $\rho(n)$-approximation of LABELED Min PM becomes a $2 \rho(n)$-approximation of LABELED $\operatorname{Min} P M_{1}$, and conversely a $\rho(n)$-approximation of LABELED $M i n P M_{1}$ remains a $\rho(n)$-approximation of LABELED Min PM. Actually, since LABELED Min PM is simple, [8] (i.e., the restriction to $o p t(I) \leq k$ is polynomial), we can see that LABELED Min PM and LABELED $\operatorname{Min} P M_{1}$ are asymptotically equivalent to approximate. Hence, the proposed results for LABELED Min $P M_{1}$ also hold for LABELED Min PM.

\section{A self improving operation on some classes of graphs}

We now propose a self improving operation for some classes of instances $\mathcal{P}_{k}$ described as follows. $I=(H, \mathcal{L}) \in \mathcal{P}_{k}$ where $H=(V, E)$ if and only if the following properties are satisfied:

(i) $H$ is planar of maximum degree $k$ and connected.

(ii) $\exists u, v \in V$ such that $\left[u, u_{1}\right]$ and $\left[v, v_{1}\right]$ for some $u_{1}, v_{1} \in V$ are the only edges incident to $u$ and $v$ (the vertices $u$ and $v$ will be called the extreme vertices of $H$ ). Moreover, these two edges have color $c_{0}$, i.e., $\mathcal{L}\left(\left[u, u_{1}\right]\right)=\mathcal{L}\left(\left[v, v_{1}\right]\right)=c_{0}$.

(iii) $H$ is bipartite and admits a perfect matching (in particular, $H$ has an even number of vertices).

(iv) $H\left[\left\{c_{0}\right\}\right]=\left(V, \mathcal{L}_{0}\right)$, the subgraph induced by edges of color $c_{0}$ does not have any perfect matching and the subgraph $H\left[\mathcal{L}(E) \backslash\left\{c_{0}\right\}\right]$ induced by edges of colors different from $c_{0}$ is acyclic. 
(v) if $H^{\prime}=H \backslash\{u, v\}$ denotes the subgraph induced by $V \backslash\{u, v\}$, then $H^{\prime}\left[\left\{c_{0}\right\}\right]=$ $\left(V \backslash\{u, v\}, \mathcal{L}_{0}\right)$ has a perfect matching denoted by $M_{c_{0}}$.

We have $\mathcal{P}_{1}=\emptyset$ and $\mathcal{P}_{2}$ is the set of odd paths from $u$ to $v$ alternating matchings $M$ and $M_{c_{0}}$ where $M_{c_{0}}$ is only colored by color $c_{0}$. Finally, we define the class $\mathcal{P}_{*}$ by $\mathcal{P}_{*}=\cup_{k} \mathcal{P}_{k}$.

Restricted label squaring operation. Given an instance $I=(H, \mathcal{L}) \in \mathcal{P}_{k}$ of LABELED Min $P M$, its label squaring instance is $I^{2}=\left(H^{2}, \mathcal{L}^{2}\right)$ with $H^{2}=\left(V^{2}, E^{2}\right)$, where

1. The graph $H^{2}$ is created by removing each edge $e=[x, y]$ of $H$ with color different from $c_{0}$ and placing instead of it a copy $H(e)$ of $H$, such that $x$ and $y$ are now identified with $u$ and $v$ of $H(e)$, respectively.

2. For each copy $H(e)$ of $H$ and for an edge $e^{\prime}$ in $H(e)$ with color different from $c_{0}$, the new color of $e^{\prime}$ is $\mathcal{L}^{2}\left(e^{\prime}\right)=\left(\mathcal{L}(e), \mathcal{L}\left(e^{\prime}\right)\right)$. The remaining edges of copy $H(e)$ keep their color $c_{0}$, that is if $\mathcal{L}\left(e^{\prime}\right)=c_{0}$, then $\mathcal{L}^{2}\left(e^{\prime}\right)=c_{0}$.

Let us prove that classes $\mathcal{P}_{k}$ are closed under restricted label squaring operation.

Lemma 2.1 If $I \in \mathcal{P}_{k}$, then $I^{2} \in \mathcal{P}_{k}$.

Proof: Let $I \in \mathcal{P}_{k}$. The proofs of $(i)$ and $(i i)$ are obvious since $u$ and $v$ have degree 1 .

For (iii), since $H$ and $H \backslash\{u, v\}$ admit a perfect matching, we deduce that $u \in L$ and $v \in R$ where $(L, R)$ is the bipartition of $H$. Thus, we can extend the bipartition to $H^{2}$ by taking for each $H(e)$ a copy of the bipartition. Finally, it is easy to verify that $H^{2}$ admits a perfect matching if $H$ does. Actually, given a perfect matching $M$ of $H$, a perfect matching $M^{2}$ of $H^{2}$ can be constructed as follows: for any edge $e$ of $H$ with a color different from $c_{0}$, if $e \in M$, then take for $H(e)$ a copy of $M$; if $e \notin M$, then take for $H(e) \backslash\{u, v\}$ a copy of a perfect matching of $H \backslash\{u, v\}$ of color $c_{0}$. Moreover, given any edge $e$ in $H$ with color $c_{0}$, then $e \in M^{2}$ iff $e \in M$.

For (iv) assume on the contrary that is $H^{2}\left[\left\{c_{0}\right\}\right]$ admits a perfect matching $M$ and $H\left[\left\{c_{0}\right\}\right]$ does not. By hypothesis, in each copy $H([x, y])$, the vertices $x$ and $y$ are not saturated by $M$ because some vertices (in fact, an even positive number of vertices) of $H([x, y])$ are not saturated by $M$ (otherwise, the restriction of $M$ to $H([x, y]$ ) is a perfect matching for $H)$, and $x$ and $y$ are the only vertices of $H([x, y])$ that do not need to be saturated by $M$ (if other vertices are not saturated by $M$ in $H([x, y]$ ), then $M$ is not a perfect matching for $H^{2}$ ). Hence, the edges of $M$ which do not belong to $H(e)$ form a perfect matching of $H\left[\left\{c_{0}\right\}\right]$, contradiction. Moreover, using Property (ii), it is easy to verify that the subgraph $H^{2}\left[\mathcal{L}^{2}\left(E^{2}\right) \backslash\left\{c_{0}\right\}\right]$ is acyclic whenever $H\left[\mathcal{L}(E) \backslash\left\{c_{0}\right\}\right]$ is acyclic.

For $(v)$ let $M_{c_{0}}$ be a perfect matching of $H^{\prime}=H \backslash\{u, v\}$ only using color $c_{0}$. We complete $M_{c_{0}}$ by taking for each copy $H(e)$ a copy of $M_{c_{0}}$. In this way, we obtain a perfect matching of $H^{2} \backslash\{u, v\}$ that uses color $c_{0}$ only.

We now propose an approximation preserving reduction using the label squaring operation on $\mathcal{P}_{k}$.

Theorem 2.2 Let $I=(H, \mathcal{L}) \in \mathcal{P}_{k}$. Any solution of $I^{2}$ with value apx $\left(I^{2}\right) \leq \rho \operatorname{opt}\left(I^{2}\right)$ for LABELED Min $P M_{1}$, can be polynomially converted into a solution of I for LABELED Min $P M_{1}$ with a value apx $(I) \leq \sqrt{\rho}$ opt $(I)$. 
Proof: Let $M^{*}$ be an optimal perfect matching of $I \in \mathcal{P}_{k}$ and let $\mathcal{L}^{*}$ be the set of colors used by $M^{*}$ (we have $\operatorname{opt}(I)=\left|\mathcal{L}^{*}\right|$ ). We construct a perfect matching $M^{2}$ for $H^{2}$ as follows. For each edge $e$ of $H$, we do the following. If $\mathcal{L}(e) \neq c_{0}$ and $e \in M^{*}$, then we take for $H(e)$ a copy of $M^{*}$ using colors $(\mathcal{L}(e), l)$ for $l \in \mathcal{L}^{*}$ and color $c_{0}$. If $\mathcal{L}(e) \neq c_{0}$ and $e \notin M^{*}$, then we take for $H(e) \backslash\{u, v\}$ a perfect matching for $H \backslash\{u, v\}$ of color $c_{0}$. If $\mathcal{L}(e)=c_{0}$, then $e \in M^{2}$ iff $e \in M$. This matching uses $(\operatorname{opt}(I)-1)^{2}+1$ colors and thus

$$
\operatorname{opt}_{1}\left(I^{2}\right) \leq o p t_{1}^{2}(I)
$$

Now, consider an approximate perfect matching $M^{2}$ of $H^{2}$ with value $\operatorname{apx}\left(I^{2}\right)$ and let $H\left(e_{1}\right), \ldots, H\left(e_{p}\right)$ be the copies of $H$ such that the restriction of $M^{2}$ to $H\left(e_{i}\right)$ is a perfect matching. Hence, we may always assume that $M^{2} \backslash\left(\cup_{i=1}^{p} H\left(e_{i}\right)\right)$ only uses color $c_{0}$ Because of Property $(v)$ and the fact that, in each copy $H(e)$ with $e \notin\left\{e_{1}, \ldots, e_{p}\right\}$, there is an even number of vertices (from Property $(i i i)$ ) and so neither $u$ nor $v$ is saturated. Therefore, if we denote $\mathcal{L}^{\prime}=\left\{\mathcal{L}\left(e_{i}\right): i=1, \ldots, p\right\}$ the set of colors of these edges in $H$, then for any $c_{j} \in \mathcal{L}^{\prime}$ there exists a perfect matching $M_{c_{j}, k} \subseteq M^{2}$ in copy $H\left(e_{k}\right)$ with $\mathcal{L}\left(e_{k}\right)=c_{j}$. Let $M_{c_{j}}$ be one of these perfect matchings of $H$, one that minimizes $\left|\mathcal{L}\left(M_{c_{j}, k}\right)\right|$ for any $c_{j} \in \mathcal{L}^{\prime}$, where $\left|\mathcal{L}\left(M_{c_{j}, k}\right)\right|$ is the number of colors used by $M_{c_{j}, k}$ and let $M_{0}$ be a perfect matching of $H$ containing edges $\left\{e_{1}, \ldots, e_{p}\right\}$ and all the other edges having color $c_{0}$.

The approximate perfect matching $M$ of $I$ will be given by one of the matchings $M_{c_{j}}$ or $M_{0}$ with value $\operatorname{apx}(I)=\min \left\{\left|\mathcal{L}\left(M_{0}\right)\right|,\left|\mathcal{L}\left(M_{c_{j}}\right)\right|: c_{j} \in \mathcal{L}^{\prime}\right\}$. Thus, we deduce that $\operatorname{apx}_{1}(I)=\operatorname{apx}(I)-1=\min \left\{\left|\mathcal{L}\left(M_{0}\right)\right|-1,\left|\mathcal{L}\left(M_{c_{j}}\right)\right|-1: c_{j} \in \mathcal{L}^{\prime}\right\}$ and hence:

$$
a p x_{1}^{2}(I) \leq\left(\left|\mathcal{L}\left(M_{0}\right)\right|-1\right) \min \left\{\left|\mathcal{L}\left(M_{c_{j}}\right)\right|-1: c_{j} \in \mathcal{L}^{\prime}\right\} \leq \sum_{c_{j} \in \mathcal{L}^{\prime}}\left(\left|\mathcal{L}\left(M_{c_{j}}\right)\right|-1\right) \leq a p x_{1}\left(I^{2}\right)
$$

The last inequality is valid because for $c_{j_{1}} \neq c_{j_{2}}$, the colors used in $M_{c_{j_{1}}}$ are different (except for $c_{0}$ ) from the ones used in $M_{c_{j_{2}}}$. Applying inequality (2) with an optimal perfect matching $M^{2}$ of $H^{2}$, we obtain $o p t_{1}^{2}(I) \leq o p t_{1}\left(I^{2}\right)$. Using inequality (1), we deduce $o p t_{1}^{2}(I)=o p t_{1}\left(I^{2}\right)$ and the expected result follows.

\section{Inapproximability results}

In [7], an inapproximability bound of $O(\log n)$ is obtained for LABELED Min PM in complete bipartite graphs via a reduction from Set Cover. A slight modification of this reduction allows us to obtain the same result for instances in $\mathcal{P}_{*}$.

Theorem 3.1 LABELED Min $P M_{1}$ is not $c \log n$ approximable for some constant $c>0$ for instances in $\mathcal{P}_{*}$ having $2 n$ vertices, unless $\boldsymbol{P}=\boldsymbol{N P}$.

Proof: Given a family $\mathcal{S}=\left\{S_{1}, \ldots, S_{n_{0}}\right\}$ of subsets of a ground set $X_{0}=\left\{x_{1}, \ldots, x_{m_{0}}\right\}$ (we assume that $\cup_{i=1}^{n_{0}} S_{i}=X_{0}$ ), a set cover of $X_{0}$ is a sub-family $\mathcal{S}^{\prime}=\left\{S_{f(1)}, \ldots, S_{f(p)}\right\} \subseteq$ $\mathcal{S}$ such that $\cup_{i=1}^{p} S_{f(i)}=X_{0}$; MinSC is the problem of determining a minimum-size set cover $\mathcal{S}^{*}=\left\{S_{f^{*}(1)}, \ldots, S_{f^{*}(q)}\right\}$ of $X_{0}$. Given an instance $I_{0}=\left(\mathcal{S}, X_{0}\right)$ of MinSC, its characteristic graph $G_{I_{0}}=\left(L_{0}, R_{0} ; E_{I_{0}}\right)$ is a bipartite graph with a left set $L_{0}=\left\{l_{1}, \ldots, l_{n_{0}}\right\}$ that represents the members of the family $\mathcal{S}$ and a right set $R_{0}=\left\{r_{1}, \ldots, r_{m_{0}}\right\}$ that 


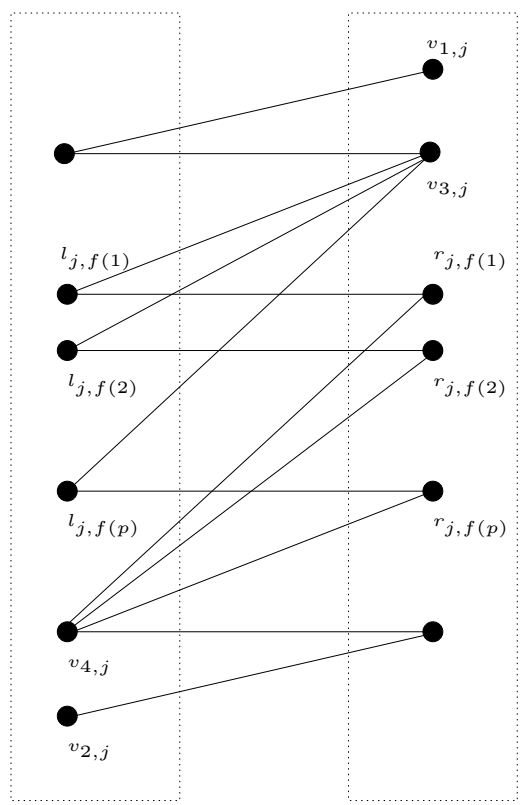

Figure 1: The gadget $H\left(x_{j}\right)$.

represents the elements of the ground set $X$; the edge-set $E_{I_{0}}$ of the characteristic graph is defined by $E_{I_{0}}=\left\{\left[l_{i}, r_{j}\right]: x_{j} \in S_{i}\right\}$.

From $I_{0}$, we construct the instance $I=(H, \mathcal{L})$ of LABELED Min PM containing $\left(n_{0}+1\right)$ colors $\left\{c_{0}, c_{1}, \ldots, c_{n_{0}}\right\}$, described as follows:

- For each element $x_{j} \in X_{0}$, we build a gadget $H\left(x_{j}\right)$ that consists of a bipartite graph of $2\left(d_{G_{I_{0}}}\left(r_{j}\right)+3\right)$ vertices and $3 d_{G_{I_{0}}}\left(r_{j}\right)+4$ edges, where $d_{G_{I_{0}}}\left(r_{j}\right)$ denotes the degree of vertex $r_{j} \in R$ in $G_{I_{0}}$. The graph $H\left(x_{j}\right)$ is illustrated in Figure 1 where the vertices $\left\{l_{f(1)}, \ldots, l_{f(p)}\right\}$ are the neighbors of $r_{j}$ in $G_{I_{0}}$ and $p=d_{G_{I_{0}}}\left(r_{j}\right)$.

- A distinct color is associated to each subset of $\mathcal{S}$. More precisely, assume that vertices $\left\{l_{f(1)}, \ldots, l_{f(p)}\right\}$ with $p=d_{G_{I_{0}}}\left(r_{j}\right)$ are the neighbors of $r_{j}$ in $G_{I_{0}}$, then color $H\left(x_{j}\right)$ as follows: for any $k=1, \ldots, p, \mathcal{L}\left(v_{3, j}, l_{j, f(k)}\right)=c_{f(k)}$ and the other edges receive color $c_{0}$.

- We complete $H=\cup_{x_{j} \in X} H\left(x_{j}\right)$ by adding edges $\left[v_{2, j}, v_{1, j+1}\right]$ with color $c_{0}$ for $j=$ $1, \ldots, m_{0}-1$.

- Finally, we set $u=v_{1,1}$ and $v=v_{2, m_{0}}$.

Clearly, $I \in \mathcal{P}_{*}$ and has $2 n=2 \sum_{r_{j} \in R}\left(d_{G_{I_{0}}}\left(r_{j}\right)+3\right)=2\left|E_{I_{0}}\right|+6 m_{0}$ vertices.

Let $\mathcal{S}^{*}$ be an optimal set cover on $I_{0}$. We can associate to each element $x_{j}$ a subset $S_{k}$ of $\mathcal{S}^{*}$ that "covers" $x_{j}$. Then, we construct a perfect matching $M^{*}$ as follows. The restriction of $M^{*}$ to gadget $H\left(x_{j}\right)$ is a perfect matching $M_{j}:\left[l_{j, f(k)}, v_{3, j}\right] \in M_{j}$ and $\left[r_{j, f(k)}, v_{4, j}\right] \in M_{j}$ ( $S_{k}$ be the subset covering $x_{j}$ ); all the others edges of $M_{j}$ are of color $c_{0}$. Thus, the perfect matching $M^{*}$ of $I=(H, \mathcal{L})$ uses exactly $\left(\left|\mathcal{S}^{*}\right|+1\right)$ colors. Conversely, let $M$ be a perfect matching on $I$. We have the following property: in each gadget $H\left(x_{j}\right)$, the edge of this gadget which is adjacent to $v_{2, j}$ is in $M$ (this is true for $H\left(x_{m_{0}}\right)$, and this can easily be 
proved by induction for all the other gadgets). This is also the case for the edge of this gadget which is adjacent to $v_{1, j}$. This implies that, in each gadget $H\left(x_{j}\right)$, there is exactly one edge of color different from $c_{0}$ that belongs to $M$. We include the subset $S_{k}$ associated with this edge in the set cover. In conclusion, the subset $\mathcal{S}^{\prime}=\left\{S_{k}: c_{k} \in \mathcal{L}(M)\right\}$ of $\mathcal{S}$ is a set cover of $X$ using $(|\mathcal{L}(M)|-1)$ sets.

Now, it is well known that the set cover problem is NP-hard to approximate within factor $c \log n_{0}$ for some constant $c>0,[2]$. This result also applies to instances $(X, \mathcal{S})$ when $|X|$ and $|\mathcal{S}|$ are polynomially related (i.e., $|X|^{q} \leq|\mathcal{S}| \leq|X|^{p}$ for some constants $p, q$ ).

Hence, given such an instance $I_{0}=(X, \mathcal{S})$, from any algorithm A solving LABELED Min $P M_{1}$ within a performance ratio $\rho_{\mathrm{A}}(I) \leq \frac{c}{q+1} \times \log (n)$ for a bipartite graph on $2 n$ vertices, we can deduce an algorithm for MinSC that guarantees the performance ratio $c \frac{1}{q+1} \log (n) \leq c \frac{1}{q+1} \log \left(n_{0}^{q+1}\right)=c \log \left(n_{0}\right)$, a contradiction.

Starting from the APX-completeness result for the vertex cover problem in cubic graphs [1], we are able to obtain the following result.

Corollary 3.2 LABeled Min $P M_{1}$ for instances in $\mathcal{P}_{3}$ is not in $\boldsymbol{P T A} \boldsymbol{S}$, unless $\boldsymbol{P}=\boldsymbol{N P}$.

Proof: Starting from the restriction of set cover where each element $x_{i}$ is covered by exactly two sets (this case is usually called vertex cover where the sets and elements can respectively be viewed as vertices and edges), we apply the same proof as in Theorem 3.1. The instance $I$ becomes an element of $\mathcal{P}_{3}$, and using for instance the APX-completeness result of [1], the expected result follows.

By applying the well known method of self improving, we obtain the two following results:

Theorem 3.3 LABELED Min $P M_{1}$ for instances in $\mathcal{P}_{3}$ is not in $\boldsymbol{A P X}$, unless $\boldsymbol{P}=\boldsymbol{N P}$.

Proof: Assume the reverse and let A be a polynomial algorithm solving LABELED Min PM within a constant performance ratio $\rho$. Let $\varepsilon>0$ (with $\varepsilon<\rho-1$ ) and choose the smallest integer $q$ such that:

$$
q \geq-\log _{2} \log _{\rho}(1+\varepsilon)
$$

Consider now an instance $I=(H, \mathcal{L}) \in \mathcal{P}_{3}$ and use the restricted label squaring operation on $I$. We produce the instance $I^{2}=\left(H^{2}, \mathcal{L}^{2}\right)$ and by repeating $q$ times this operation on $I^{2}$, we obtain thanks to Lemma 2.1 the instance $I^{2^{q}}=\left(H^{2^{q}}, \mathcal{L}^{2^{q}}\right) \in \mathcal{P}_{3}$, in time $P(|I|)$ for some polynomial $P$ since on the one hand, $I^{2}$ is obtained from $I$ in time $O\left(|I|^{2}\right)$ (we have $\left|V\left(H^{2}\right)\right|=O\left(|V(H)|^{2}\right)$ and $\left|\mathcal{L}^{2}\left(E\left(H^{2}\right)\right)\right|=O\left(|\mathcal{L}(E(H))|^{2}\right)$ ) and on the other hand, we repeat this operation a constant number of times. Using Theorem 2.2, from the $\rho$-approximation on $I^{2^{q}}$ given by A, we obtain a $\rho^{2^{-q}}$-approximation on $I$. Thanks to inequality (3), we deduce $\rho^{2^{-q}} \leq 1+\varepsilon$. Hence, we obtain a polynomial time approximation scheme for instances in $\mathcal{P}_{3}$, a contradiction with Corollary 3.2 .

Theorem 3.4 For any $\varepsilon>0$ LABELED Min $P M_{1}$ is not $2^{O\left(\log ^{1-\varepsilon} n\right)}$-approximable for instances in $\mathcal{P}_{3}$ on $n$ vertices, unless $\boldsymbol{N P} \subseteq \boldsymbol{D T I M E}\left(2^{O\left(\log ^{1 / \varepsilon} n\right)}\right)$. 
Proof: Let $\varepsilon>0$ and $I=(H, \mathcal{L}) \in \mathcal{P}_{3}$ where $H$ has $n$ vertices. Choose the smallest integer $p$ such that $n^{2^{p}} \geq 2^{\log ^{1 / \varepsilon} n}$. Thus, $2^{2^{p} \times \log n} \geq 2^{\log ^{1 / \varepsilon} n}$ and then,

$$
2^{p \times \varepsilon} \geq \log ^{1-\varepsilon} n
$$

Using the restricted label squaring operation on $I$, we produce the instance $I^{2}=$ $\left(H^{2}, \mathcal{L}^{2}\right)$. By repeating $p$ times this operation on $I^{2}$, we obtain the instance $I^{2^{p}}=\left(H^{2^{p}}, \mathcal{L}^{2^{p}}\right) \in$ $\mathcal{P}_{3}$. Since $H$ has $n$ vertices, we derive that the number $n^{\prime}$ of vertices of $H^{2^{p}}$ and the number $\left|\mathcal{L}^{2^{p}}\left(E\left(H^{2^{p}}\right)\right)\right|$ of colors of $H^{2^{p}}$ satisfy:

$$
n^{\prime} \leq n^{2^{p}} \text { and }\left|\mathcal{L}^{2^{p}}\left(E\left(H^{2^{p}}\right)\right)\right| \leq|\mathcal{L}(E(H))|^{2^{p}}
$$

Now, assume that we have a $f\left(n^{\prime}\right)$-approximation on $I^{2^{p}}$ where $f\left(n^{\prime}\right) \leq 2^{c \times \log ^{1-\varepsilon}} n^{\prime}$ for some $c>0$. Using Theorem 2.2, we obtain a $f\left(n^{\prime}\right)^{2^{-p}}$-approximation on $I$. Using inequalities (4) and (5), we deduce:

$$
\begin{aligned}
\operatorname{apx}_{1}(I) & \leq f\left(n^{\prime}\right)^{2^{-p}} \operatorname{opt}_{1}(I) \\
& \leq 2^{c \times \frac{\log 1-\varepsilon}{2^{p}} n^{\prime}} \operatorname{opt}_{1}(I) \\
& \leq 2^{c \times \frac{\log ^{1-\varepsilon} n}{2^{\varepsilon \times p}}}{ } \operatorname{opt}_{1}(I) \\
& \leq 2^{c} \operatorname{opt}_{1}(I)
\end{aligned}
$$

By definition of $p, n^{2^{p-1}}<2^{\log ^{1 / \varepsilon} n}$, and thus $n^{\prime} \leq n^{2^{p}}<2^{2 \log ^{1 / \varepsilon} n}$. In conclusion, using inequality (5), we obtain a constant approximation for $I$ in time $p o l y\left(n^{\prime}\right)=2^{O\left(\log ^{1 / \varepsilon} n\right)}$, and thus, a contradiction to Theorem 3.3.

It is natural to ask the question whether the problem is easier in cubic bipartite graphs. Here, we prove that the answer is negative.

Theorem 3.5 LABeled Min $P M_{1}$ is not in $\boldsymbol{A P X}$ in connected planar cubic bipartite graphs, unless $\boldsymbol{P}=\boldsymbol{N P}$.

Proof: The proof consists of two steps. First, using a reduction quite similar to the one of Corollary 3.2, we prove that Theorem 3.4 also holds for the sub-family $\mathcal{P}_{3}^{\prime}$ of $\mathcal{P}_{3}$ where each vertex has a degree 3 , except $u$ and $v$. Then, we transform any instance of $\mathcal{P}_{3}^{\prime}$ into a connected planar cubic bipartite graph.

Let us prove the first point. Let $G=(V, E)$ with $V=\left\{v_{1}, \ldots, v_{n}\right\}$ and $E=\left\{e_{1}, \ldots, e_{n}\right\}$ be an instance of vertex cover. We associate to any edge $e_{j}=[x, y]$ a gadget $H\left(e_{j}\right)$ described in Figure 2. All edges of $H\left(e_{j}\right)$, except $\left[v_{3, j}, l_{j, x}\right]$ and $\left[v_{3, j}, l_{j, y}\right]$ have color $c_{0}$. We have $\mathcal{L}\left(\left[v_{3, j}, l_{j, x}\right]\right)=c_{x}$ and $\mathcal{L}\left(\left[v_{3, j}, l_{j, y}\right]\right)=c_{y}$. Finally, $H\left(e_{j}\right)$ is linked to $H\left(e_{j+1}\right)$ using the graph depicted in Figure 3 where each edge is colored with $c_{0}$. We conclude this part of the proof by using arguments similar to ones given in the proof of Corollary 3.2 (the gadgets considered in both reductions having the same basic properties). Clearly, LABELEd $\operatorname{Min} P M_{1}$ is APX-hard in class $\mathcal{P}_{3}^{\prime}$. Since the restricted label squaring operation also preserves the membership in $\mathcal{P}_{3}^{\prime}$, we deduce that LABELED Min $P M_{1}$ is not in APX when the instances are restricted to $\mathcal{P}_{3}^{\prime}$.

Now, let us prove the second point. Given $I \in \mathcal{P}_{3}^{\prime}$ with $I=(G, \mathcal{L})$, we consider the instance $I^{\prime}$ where $G$ is duplicated 3 times into $G_{1}, G_{2}, G_{3}$. If $u_{i}, v_{i}$ denote the extreme 


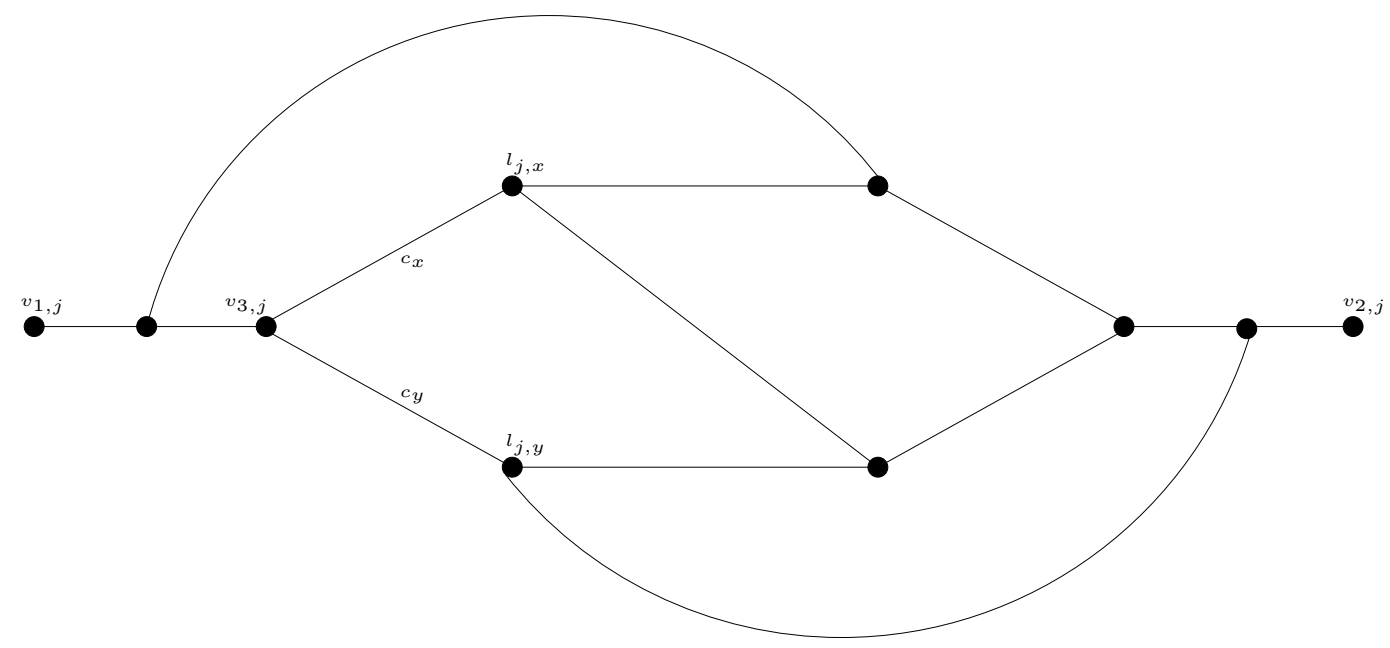

Figure 2: The gadget $H\left(e_{j}\right)$ for $e_{j}=[x, y]$.

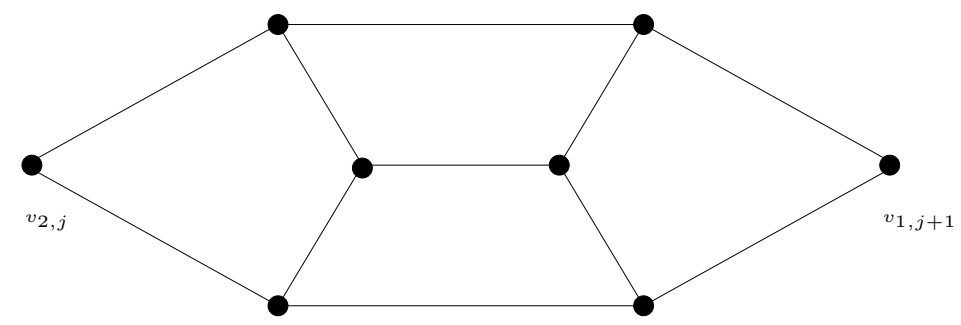

Figure 3: The gadget linking $H\left(e_{j}\right)$ to $H\left(e_{j+1}\right)$.

vertices of $G_{i}$, we shrink vertices $u_{1}, u_{2}, u_{3}$ into $u$ and $v_{1}, v_{2}, v_{3}$ into $v$. Clearly, this new graph $G^{\prime}$ is connected bipartite, planar and cubic. Given any perfect matching $M^{\prime}$ of $G^{\prime}$, the two edges of color $c_{0}$ adjacent to $u$ and $v$ in $M^{\prime}$ necessarily lie in the same copy of $G$, because there is an even number of vertices in each copy. Finally, since we can restrict ourselves to perfect matchings $M^{\prime}$ of $G^{\prime}$ that use only color $c_{0}$ for exactly two copies of $G$, the result follows.

Obviously, a proof similar to the one given in Theorem 3.4 can be applied to the Theorem 3.5 leading to the following conclusion.

Corollary 3.6 For any $\varepsilon>0$ LABELED Min $P M_{1}$ is not $2^{O\left(\log ^{1-\varepsilon} n\right)}$-approximable in connected planar cubic bipartite graphs on $n$ vertices, unless $\boldsymbol{N P} \subseteq \boldsymbol{D T I M E}\left(2^{O\left(\log ^{1 / \varepsilon} n\right)}\right)$.

Dealing with the unbounded degree case (that is instances of $\mathcal{P}_{*}$ ), we can deduce the following stronger result:

Theorem 3.7 LABELED Min $P M_{1}$ for instances in $\mathcal{P}_{*}$ is not in polyLog-APX, unless $P=N P$.

Proof: Assume on the contrary that LABELED Min $P M_{1}$ is $f(n)$-approximable with $f(n) \leq$ $c \log ^{k} n$ for some constants $c>0$ and $k \geq 1$. Let $I=(H, \mathcal{L}) \in \mathcal{P}_{*}$ where $H$ has $2 n$ vertices. 
Let $p=\lceil\log k\rceil+1$. Using as previously $2^{p}$ times the restricted label squaring operation on $I$, we produce in polynomial time the instance $I^{2^{p}}=\left(H^{2^{p}}, \mathcal{L}^{2^{p}}\right) \in \mathcal{P}_{*}$. The same arguments as in Theorem 3.4 allow us to obtain a contradiction with Theorem 3.1 since from Property (iv) of Lemma 2.1, the inequality (5) also holds.

Acknowledgments. The author wants to thank Bruno Escoffier and Laurent Gourvès for useful comments on earlier versions of this paper.

\section{References}

[1] P. Alimonti and V. Kann, "Hardness of approximating problems on cubic graphs," Proc. CIAC'97, LNCS 1203 (1997) 288-298.

[2] S. Arora and M. Sudan, "Improved Low-Degree Testing and its Applications," Combinatorica, 23, (2003), 365-426.

[3] K. Cameron, "Coloured matchings in bipartite graphs," Discrete Mathematics, 169 (1997) 205-209.

[4] M. Costa, D. de Werra, C. Picouleau, and B. Ries, "Bicolored matchings in some classes of graphs," Graphs and Combinatorics, 23 (2007) 47-60.

[5] A. Itai, M. Rodeh, and S. Tanimoto, "Some matching problems in bipartite graphs," J. ACM, 25 (1978) 517-525.

[6] J. Monnot, " On Complexity and Approximability of the Labeled Maximum/Perfect Matching Problems," Proc. ISAAC'05, LNCS 3827 (2005) 934-943.

[7] J. Monnot, "The Labeled perfect matching in bipartite graphs," Information Processing Letters 96 (2005) 81-88.

[8] A. Paz and S. Moran, "Non deterministic polynomial optimisation problems and their approximation," Theoretical Computer Science, 95 (1981) 251-277.

[9] M. B. Richey and A. P. Punnen, " Minimum Perfect Bipartite Matchings and Spanning Trees under Categorization," Discrete Applied Mathematics 39 (1992) 147-153. 